18

\title{
Luminescence of Single Semiconductor Nanocrystals at Room Temperature as Observed with Confocal Microscopy*
}

(C) V. Zakharov ${ }^{1,2}$, M. Stepanova ${ }^{1}$, M. Baranov ${ }^{1}$, A. Dubavik $^{1}$, T. Kormilina ${ }^{1}$,

S. Cherevkov ${ }^{1}$, L. Borodina ${ }^{1}$, and A. Veniaminov ${ }^{1}$

${ }^{1}$ ITMO University,

197101 St. Petersburg, Russia

${ }^{2}$ Optec LLC,

197101 St. Petersburg, Russia

e-mail: viktor-zah@yandex.ru

Received July 6, 2018

Luminescent images of a few distinguishable alloyed semiconductor quantum dots obtained at room temperature using confocal scanning microscopy with spectral and temporal resolution demonstrate blinking of spatially distinguishable spots and local luminescence spectra varying within the ensemble.

DOI: $10.21883 /$ OS.2018.11.46841.214-18

\footnotetext{
* International Conference „PCNSPA 2018 - Photonic Colloidal Nanostructures: Synthesis, Properties, and Applications" ${ }^{\text {“ }}$, Saint Petersburg, Russia, June 4-8, 2018.

Полный текст статьи опубликован в английской версии журнала.
} 